\title{
Use of dual rf systems to accelerate large longitudinal emittance intense beam in a synchrotron
}

\author{
C. M. Bhat, ${ }^{*}$ J. A. MacLachlan, and V. Wu ${ }^{\dagger}$ \\ Fermi National Accelerator Laboratory, P.O. Box 500, Batavia, Illinois 60510, USA
}

(Received 7 December 2006; published 30 March 2007)

\begin{abstract}
Preserving the beam brightness and emittance during acceleration for high energy collider operation is a long-standing challenge for synchrotron injectors. Radio-frequency manipulation schemes used to produce bright bunches in these synchrotrons are often responsible for both beam loss and for the emittance dilution; the impact of such effects becomes the main obstacle to improve the collider luminosity. We have developed a scheme for acceleration of intense beam bunches of large longitudinal emittance using two rf systems. The development of such a method primarily came about to improve the overall injection to extraction efficiency of the antiproton beam in the Main Injector used for the protonantiproton collider operation of the Tevatron at Fermilab. This technique is quite general and can be applied at other facilities. Multiparticle beam dynamics simulations of the scheme have shown that one can eliminate the beam loss and minimize the emittance growth. The scheme has been demonstrated with beam experiments in the Main Injector using the 2.5 and $53 \mathrm{MHz}$ rf systems with harmonic ratio of 1:21.
\end{abstract}

DOI: 10.1103/PhysRevSTAB.10.034403

PACS numbers: 29.27.Bd, 29.20.Lq, 29.27.Fh

\section{INTRODUCTION}

Producing low emittance bright beam bunches and preserving their properties during acceleration in high energy injector synchrotrons has been one of the major challenges for hadron colliders [1-3]. The lower limit on the emittance of a bunch is set by the particle beam source. Quite often, several bunches of beam particles with low emittance are added together to form a more intense bunch at some intermediate energy by rf manipulation in one of the preaccelerators prior to the collider synchrotron. These rf manipulations have become the main source of longitudinal emittance dilution and in some cases significant beam loss. In the case of the proton-antiproton collider at Fermilab, the currently adopted antiproton beam manipulation scheme [2,4-6] in the Main Injector [7] is responsible for beam loss and emittance growth prior to injection into the Tevatron. Further, if the longitudinal emittance ${ }^{1}$ $\left(\varepsilon_{l}\right)$ [8] of the beam is larger than the acceptance of the Tevatron during acceleration from 150 to $980 \mathrm{GeV}$, the particles outside the acceptance range are lost. This is not only detrimental for antiproton beam economy but also limits the collider luminosity.

The immediate goal of the Tevatron proton-antiproton collider at Fermilab is to maximize the integrated luminosity delivered to the collider experiments at $\mathrm{CDF}$ and $\mathrm{D} 0$ in the period before the LHC [9] at CERN is turned on later in the decade. Fermilab has developed run II upgrade plans [10] aimed at luminosity improvement and is currently

\footnotetext{
*Electronic address: cbhat@fnal.gov

${ }^{\dagger}$ Current address: Department of Radiation Oncology, UCSF Comprehensive Cancer Center, 1600 Divisadero St., San Francisco, CA 94143, USA.

${ }^{1}$ The italicized word that appears in the paper is specific to beam physics. We use them in the rest of the text without defining.
}

implementing them in stages. Consequently, the peak luminosity has been raised significantly [11], and a new world record for hadron collider peak luminosity has been achieved (current peak luminosity at the Tevatron is about $50 \%$ greater than the ISR record at CERN). Another $20 \%$ increase in peak luminosity is required to meet the run II design goals. The peak luminosity at a collider is given by [8]

$$
L=\frac{N_{p} N_{a} B f_{\mathrm{Tev}}\left(6 \beta_{\mathrm{Tev}} \gamma_{\mathrm{Tev}}\right)}{2 \pi \beta^{*}\left(\varepsilon_{p}+\varepsilon_{a}\right)} H\left(\frac{\beta^{*}}{\sqrt{\frac{1}{2}\left(\sigma_{p}^{2}+\sigma_{a}^{2}\right)}}\right),
$$

where $N_{p}$ and $N_{a}$ are the number of protons and antiprotons per bunch, respectively. $B$ is the number of bunches of each type. $f_{\mathrm{Tev}}, \beta_{\mathrm{Tev}}$ and $\gamma_{\mathrm{Tev}}$ are the revolution frequency, relativistic velocity, and the energy of the beam, respectively. $\beta^{*}$ is the common value lattice functions $\beta_{x}$ and $\beta_{y}$ at the interaction points, $\varepsilon$ is the normalized transverse emittance. $H$ is the hourglass factor, which depends on proton and antiproton root mean square bunch lengths $\sigma_{p}$, $\sigma_{a}$ and crossing angles at the interaction points. For high luminosity it is important to preserve the beam brightness of both the proton beam and the antiproton beam in the Tevatron and through the upstream injector accelerator chain. At present, the peak and integrated luminosities at the Tevatron are limited significantly by the availability of $9 \mathrm{GeV}$ antiprotons. At Fermilab they are produced in high energy collisions of proton beam on a solid target. They are stored and cooled in the antiproton Accumulator ring [1] and the Recycler [12] at $9 \mathrm{GeV}$.

Currently, the final antiproton bunches required for the Tevatron are generated by a multibunch coalescing process at $150 \mathrm{GeV}$ [4-6] in the Main Injector. In this scheme four $2.5 \mathrm{MHz}$ bunches of antiprotons are transferred from the Accumulator or the Recycler synchronously to buckets of the $2.5 \mathrm{MHz}$ rf system (harmonic number $h=28$ ) in the 
Main Injector at $9 \mathrm{GeV}$. Each bunch is subdivided into five to eleven $53 \mathrm{MHz}$ bunches. These bunches are accelerated from 9 to $150 \mathrm{GeV}$ using the $53 \mathrm{MHz}$ rf. Dividing each $2.5 \mathrm{MHz}$ bunch into several $53 \mathrm{MHz}$ bunches of smaller longitudinal emittances at $9 \mathrm{GeV}$ is essential to eliminate beam loss at transition crossing in the Main Injector for $53 \mathrm{MHz}$ acceleration. Once the bunches are at $150 \mathrm{GeV}$, each group of antiproton bunches is coalesced into one $53 \mathrm{MHz}$ bunch by rotating them for a quarter synchrotron period in a $2.5 \mathrm{MHz}$ rf bucket and recapturing them in a large $53 \mathrm{MHz}$ bucket. Thus, each $2.5 \mathrm{MHz}$ bunch undergoes two sets of rf manipulations which are potential sources of emittance dilution and beam loss. Eventually, four such bunches are injected into the Tevatron. A protonantiproton store in the Tevatron needs nine transfers of this type. Thus, there are 36 antiproton bunches per store.

The practical limitations of this scheme are (a) reduced beam particle density during the $53 \mathrm{MHz}$ division of the $2.5 \mathrm{MHz}$ bunches at $9 \mathrm{GeV}$, (b) emittance dilution during bunch coalescing (The interbunch spacing in each group of $53 \mathrm{MHz}$ antiproton bunches contributes to the longitudinal emittance dilution during coalescing. For example, for seven bunch coalescing, it can be shown that the interbunch spaces alone give rise to a minimum of $23 \%$ dilution in the longitudinal emittance of the final combined bunch.), and (c) failure to capture $10 \%$ or more of the antiprotons into the $53 \mathrm{MHz}$ buckets during coalescing.

Figure 1(a) shows the measured longitudinal emittance of the antiproton bunches at injection into the Main Injector as a function of that at extraction to the Tevatron for all of the proton-antiproton stores from June 2005 to October 2006. The data showed that the distribution of longitudinal emittances is nearly independent of bunch intensity, and the average longitudinal emittance dilution is in excess of $100 \%$. Note that the data for $\varepsilon_{l}$ at $150 \mathrm{GeV}$ shown in Fig. 1(a) include only the surviving antiprotons in the final coalescing bucket at the time of extraction; consequently, the true $\varepsilon_{l}$ of the bunch after coalescing is larger than that shown here. Figure 1(b) shows antiproton injection to extraction efficiency, combining the efficiencies for 2.5 to $53 \mathrm{MHz}$ bunch division, $53 \mathrm{MHz}$ acceleration, and coalescing. The coalescing efficiency is the ratio of bunch intensities at extraction to that at $150 \mathrm{GeV}$ before coalescing. The overall efficiency is plotted versus the average intensity of the antiproton bunch injected into the Main Injector for the same period. The data show a beam loss is inevitable and is in the range of $10 \%$ to $25 \%$. In any case, this sort of antiproton loss and longitudinal emittance dilution translates into reduced luminosity in the Tevatron [see Eq. (1)]. We believe that the current coalescing technique used in the Main Injector has approached its maximum efficiency for the present operating conditions and is likely to become less efficient at higher intensities and larger longitudinal emittances. The run II upgrade plans, however, call for antiproton intensity $\approx 17 \times 10^{10}$
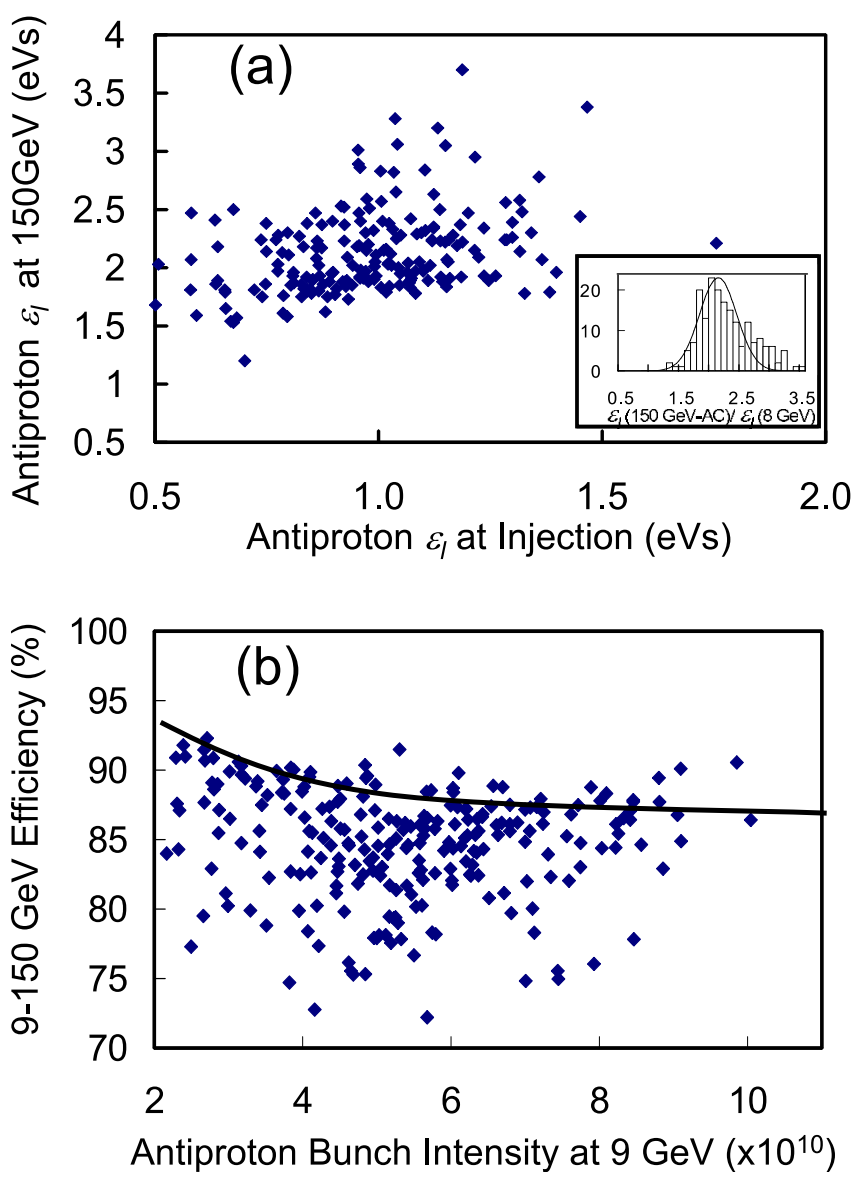

FIG. 1. (Color) (a) Measured average $\varepsilon_{l}(90 \%)$ for the antiproton bunches in the Main Injector at $150 \mathrm{GeV}$ after coalescing and before extraction to the Tevatron versus to that at injection for the current acceleration scheme in the Main Injector. (Errors on $\varepsilon_{l} \sim 15 \%$.) Each point is an average on thirty-six antiproton bunches. The inset is the histogram of the ratio of average $\varepsilon_{l}$ at $150 \mathrm{GeV}$ to that at $9 \mathrm{GeV}(2.5 \mathrm{MHz}$ bunches). (b) Measured average injection to extraction antiproton efficiency in the Main Injector versus $2.5 \mathrm{MHz}$ bunch intensity at $9 \mathrm{GeV}$. The line on (b) represents an average maximum efficiency for a given antiproton intensity at $9 \mathrm{GeV}$. For other details see the text.

antiproton/bunch at injection with $\varepsilon_{l}$ in the range of 1 $2 \mathrm{eV} \mathrm{s}$ in the future. This intensity will be about twice the maximum antiproton bunch intensity seen so far from the Accumulator or from the Recycler (as of December 2006). These problems have motivated us to develop a new technique of beam acceleration in the Main Injector.

Designing the rf systems for a medium energy synchrotron to accelerate bright and large longitudinal emittance beam bunches in a wide energy range is complex and expensive. Therefore, considerable efforts have been devoted recently to develop new rf manipulation techniques which involve multibunch coalescing without longitudinal emittance growth and subsequent acceleration [13,14]. In all cases, one requires a number of specially designed $\mathrm{rf}$ systems. Recently, a method for antiproton acceleration in the Main Injector without coalescing has been suggested 
[15] as an alternative to that described here. But this method demands that $\varepsilon_{l}<0.6 \mathrm{eV} \mathrm{s} / 2.5 \mathrm{MHz}$ bunch at $9 \mathrm{GeV}$ while our method is quite adequate for $\varepsilon_{l} \leq 3 \mathrm{eV} \mathrm{s}$.

In this paper a new scheme $[12,16]$ for acceleration of bright antiproton bunches of large longitudinal emittance is described and demonstrated using beam. The technique involves the use of two rf systems of differing harmonics of the beam circulation frequency in sequence. The first step in this scheme is to accelerate the bunches from injection energy to some intermediate energy, above the transition energy of the synchrotron using a low harmonic rf system. Then the bunches are transferred to buckets of higher harmonic rf system at a fixed energy and accelerated to the final energy. Coalescing of multiple bunches is not required. The scheme is rather general and can be used for deceleration $[12,17]$ of large longitudinal emittance beam bunches, and can be adopted with the available rf systems in the Main Injector. Preliminary results of multiparticle beam dynamics modeling and beam studies for acceleration have been presented previously [18].

The organization of the paper is as follows: In Sec. II we briefly review the relevant longitudinal beam dynamics, general principle of synchronous beam transfer between $\mathrm{rf}$ buckets, and the proposed acceleration scheme. Multiparticle beam dynamics simulation of the scheme including wakefield effects using ESME [19] applied to the case of the Main Injector is presented in Sec. III. In Sec. IV we describe the demonstration of the feasibility of the technique using a proton beam in the Main Injector. In the last section we make a few comments and summarize the results.

\section{LONGITUDINAL BEAM DYNAMICS IN A SYNCHROTRON AND PRINCIPLE OF HARMONIC TRANSFER}

A particle beam in a synchrotron at any given time during acceleration is characterized by longitudinal and transverse emittances. In the absence of synchro-betatron coupling these two quantities are independent. The particles with synchronous energy $E_{s}$ are accelerated each time they traverse the rf cavity gaps at a phase $0<\phi_{s}<\pi$ by an effective accelerating voltage $V\left(\phi_{s}\right)=V_{0} \sin \phi_{s}$; the subscript $s$ stands for the synchronous particle. The quantity $V_{0}$ is the peak rf voltage. A particle with energy $E=$ $E_{s}+\Delta E$ arrives at the cavity gap a little earlier than the synchronous particle; its phase is $\phi=\phi_{s}-\Delta \phi$. The rate of acceleration is given by $[8,20,21]$

$$
\dot{E}=\frac{\omega}{2 \pi} e V_{0} \sin \phi,
$$

where the angular revolution frequency of the particle is $\omega=2 \pi \beta c / C, C$ is circumference of the accelerator, and $\beta c$ is particle velocity. The particles which lie within a certain maximum phase offset form a bunch and are accelerated together along with the synchronous particle. The equation of motion for the energy difference is

$$
\frac{d}{d t}\left(\frac{\Delta E}{\omega_{s}}\right)=\frac{e V_{0}}{2 \pi}\left(\sin \phi-\sin \phi_{s}\right),
$$

and the corresponding rate of change of phase of the particle is

$$
\frac{d \Delta \phi}{d t}=-\frac{h \omega_{s}^{2} \eta}{\beta_{s}^{2} E_{s}}\left(\frac{\Delta E}{\omega_{s}}\right) .
$$

The quantity $\eta=\frac{1}{\gamma_{T}^{2}}-\frac{1}{\gamma_{s}^{2}}$ is called the phase slip factor. $\gamma_{s}$ is the ratio of synchronous energy to the rest mass energy of the particle, and $\gamma_{T}$ is the value of $\gamma_{s}$ at the transition energy. A particle trapped by the $\mathrm{rf}$ wave (i.e., in a $\mathrm{rf}$ bucket) will undergo synchrotron oscillations in $(\Delta E, \Delta \phi)$ phase space.

In the context of the present study, most rf manipulations are carried out at a fixed energy; thus $\sin \left(\phi_{s}\right)=0$ (or $\pi$ ). Then the synchrotron oscillation frequency of a particle with phase amplitude $\hat{\phi}$ is given by

$$
f_{\text {syn }}=\sqrt{\frac{h \omega_{s}^{2}}{\beta_{s}^{2}} \frac{|\eta|}{E_{s}} \frac{e V_{0}}{(2 \pi)^{3}}} \frac{\pi}{4 \int_{0}^{\pi / 2}\left[1-X \sin ^{2} x\right]^{-(1 / 2)} d x},
$$

with $X=\sin ^{2}(\hat{\phi} / 2)$. The half energy spread of the beam bunch is given by [21]

$$
\Delta \hat{E}= \pm 2 \sqrt{\frac{e V_{0} E_{s} \beta_{s}^{2} X^{2}}{2 \pi h|\eta|}} .
$$

The maximum possible energy spread with no beam loss during acceleration is a measure of the energy acceptance of the accelerator. For an elliptical distribution of particles in $(\Delta E, \Delta \phi)$ phase space, the corresponding phase-space area occupied by the particles is

$$
\varepsilon_{l}=\pi \hat{\phi} \Delta \hat{E}
$$

The bucket area encompassing the beam bunch is

$$
S_{B}=16 \sqrt{\frac{e V_{0}}{2 \pi h^{3}} \frac{\beta_{s}^{2}}{\omega_{s}^{2}} \frac{E_{s}}{|\eta|} .}
$$

\section{A. Transition crossing}

As the bunch approaches transition energy during acceleration, $\eta$ vanishes and, from Eq. (5), the synchrotron oscillation frequency goes to zero. The beam dynamics near the transition energy in a synchrotron are quite complicated and have been addressed extensively [16,22,23]. During this time the beam particles are subjected to nonadiabatic forces for a period of approximately $T_{n a}$, which is given by [24]

$$
T_{n a}= \pm\left[\frac{\beta_{T}^{2} \gamma_{T}^{4}}{2 \omega_{s} h} \frac{\left|\tan \phi_{s}\right|}{\dot{\gamma}_{T}^{2}}\right]^{1 / 3},
$$

where $\dot{\gamma}_{T}$ is the rate of change of $\gamma$ at transition energy. 
Moreover, the off-energy particles do not cross the transition energy at the same time as the synchronous particles. As a result of this, there is a period during which some particles are above the transition energy and others are below. In the absence of rf focusing, the off synchronous particles in a bunch will be subjected to a nonlinear rf force for a certain length of time given by $[21,25,26]$

$$
T_{n l}= \pm\left[\alpha_{1} \gamma_{T}^{2}+\frac{3 \beta_{T}^{2}}{2}\right] \frac{\gamma_{T}}{2 \dot{\gamma}_{T}} \frac{\Delta E}{\beta_{T}^{2} E_{T}},
$$

with $\alpha_{o} \alpha_{1}$ being the second order term in the expansion of the path length in $\frac{\Delta p}{p}=\frac{1}{\beta^{2}} \frac{\Delta E}{E}$ with momentum compaction factor $\alpha_{o}=\gamma_{T}^{-2}$ and $\alpha_{1}$ is Johnsen parameter. The sum of nonadiabatic and nonlinear time is a critical interval for transition crossing; in general this period should be minimized by the fastest possible acceleration scheme and choice of rf parameters.

If we assume for $|t|>T_{n a}$ (with $t=0$ at the transition crossing time) the particles follow the contours of synchrotron oscillation given by Eqs. (3) and (4), and for $|t|<T_{n a}$, the particles make no synchrotron oscillations, then one can write down an approximate analytical expression for the energy spread as follows [21]:

$$
(\Delta \hat{E})_{T}=0.502 \sqrt{\frac{\varepsilon_{l} \gamma_{T}^{4} E_{T} \beta_{T}^{2}}{T_{n a}^{2} \dot{\gamma}_{T}}}
$$

For a given $\varepsilon_{l}$, the $(\Delta \hat{E})_{T}$ scales as $h^{1 / 3}$ and $\dot{\gamma}_{T}^{1 / 6}$. Therefore, by decreasing $h$ and $\dot{\gamma}_{T}$, though it is a weak dependence, one can decrease the $(\Delta \hat{E})_{T}$ and make it sufficiently smaller than the accelerator energy acceptance at transition.

\section{B. Bunch transfer between two rf harmonics}

At the time of transferring a bunch from an rf bucket at one harmonic to that at another harmonic in a synchrotron, the phase-space distribution of the particles should match before and after to eliminate longitudinal emittance dilution. Such matching requires that the shape of the sinusoidal $\mathrm{rf}$ wave at the bunch center be the same. At a fixed energy this implies $V_{2} / V_{1}=h_{1} / h_{2}$; the subscripts " 1 " and " 2 " correspond to the two different rf systems with $h_{2}>h_{1}$. For the Fermilab Main Injector with rf systems at $h=28$ and 588, the matching condition calls for $V_{h=28}=$ $21 V_{h=588}$, far more than available from the current $h=28$ rf system in the Main Injector.

Another way to produce an approximately matched bunch in a higher frequency rf bucket is to reduce the voltage $V_{1}$ adiabatically to increase the bunch length and quickly rotate by a quarter rotation [16]. The adiabatic bunch lengthening generally is subject to instability induced by rf noise, particularly when energy spread of the beam is extremely small. Furthermore, in the case of an intense bunch, the beam loading poses additional problems at relatively low $V_{1}$. Therefore, the final matched distribu- tion of a bunch can be obtained by two successive quarter rotations, one with a rf voltage slightly more than

$$
V_{0}=\frac{\pi h|\eta|}{2 e E_{s} \beta_{s}^{2}}\left[\frac{\Delta \hat{E}_{\text {final }}}{X}\right]^{2}
$$

followed by the one with maximum $V_{1}$. In Eq. (12) the $\Delta \hat{E}_{\text {final }}$ corresponds to the final energy spread of the bunch at the end of the acceleration with the lower harmonic rf system. In any case, it is important to perform the rotations in the linear region of the rf wave, otherwise, significant shape mismatch occurs, leading to eventual longitudinal emittance growth. The $E_{s}$ is chosen so that for the available rf voltage on the higher harmonic rf system, the bucket area [Eq. (8)] is large enough to match the beam particle distribution after the second rotation and accelerate further. (Besides, $E_{s}$ should be sufficiently far from transition energy of the synchrotron to avoid any instability that would result from transition crossing.) Immediately after the second rotation, the bunch is transferred to the buckets of the higher harmonic rf system. Figure 2 shows a schematic view of the steps involved in matching the phasespace distribution. To illustrate the principle of the scheme, we have chosen the harmonic ratio of 1:7. The processes involved in Figs. 2(a)-2(e) are carried out with the low harmonic rf system. The rest are in the higher harmonic rf system with matched rf voltage and a phase angle of $180^{\circ}$ (the phase angle will be $0^{\circ}$ if harmonic transfer is carried out below the transition energy). All of the rf manipulations shown here are carried out at a constant energy. This method is much faster than adiabatic matching and can be carried out at relatively low rf voltage. Furthermore, the required rf voltage for the low harmonic rf system can be significantly larger than for the adiabatic bunch lengthening case explained earlier but yet large enough from the standpoint of beam loading.

For bunches with large longitudinal emittance, one might carry out two sets of similar bunch rotations in succession as explained above instead of just one. (Thus there will be a total of four quarter bunch rotations.) In principle, by this method one can get a better match to the buckets of higher harmonic rf system. However, implementation of two successive sets of rotations is quite a challenge.

In the case of the Main Injector, the available bucket area at $9 \mathrm{GeV}$ with the $h=588 \mathrm{rf}$ system is about $1.1 \mathrm{eV} \mathrm{s}$ while that for $h=28$ it is about $20 \mathrm{eV} \mathrm{s}$. Consequently, the $h=588 \mathrm{rf}$ buckets cannot be used for antiproton bunches with $\varepsilon_{l} \geq 1.1 \mathrm{eV} \mathrm{s}$, either for capture or for beam acceleration without any particle loss. Therefore, the bunches with large $\varepsilon_{l}$ are injected into $h=28$ buckets and accelerated from 9 to $27 \mathrm{GeV}$ through the transition energy of $20.49 \mathrm{GeV}$ using the standard phase jump scheme while crossing transition energy. The rate of acceleration is currently limited to $\dot{\gamma}=3.2 / \mathrm{sec}$ by the available maximum $\mathrm{rf}$ voltage of $\sim 60 \mathrm{kV}$ for the $h=28$ system, although the 


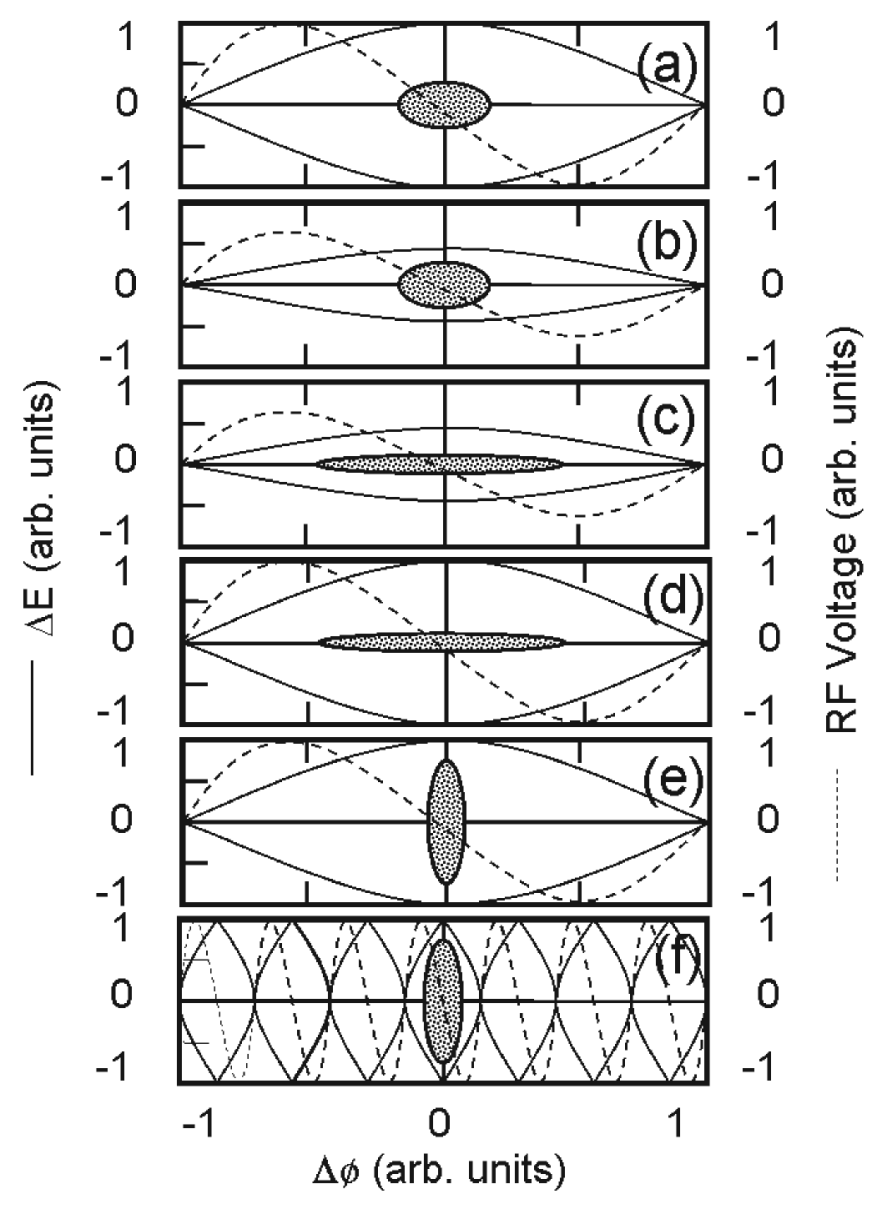

FIG. 2. Schematic view of the synchronous transfer of a bunch in a bucket of lower harmonic number to that with higher harmonic number. The solid lines and dashed lines represent the rf bucket contours and rf waveforms, respectively. $(\Delta E, \Delta \phi)$-phase distributions of beam particles (bunch shown in the middle of each bucket) shown for each stage are as follows: (a) beam particles in a bucket after the end of first acceleration (at this stage generally the $V$ is large); (b) the rf voltage is brought down quickly to a smaller value so that the bucket height is slightly larger than the beam height (bunch is not matched to bucket); (c) after a quarter synchrotron period; (d) after rapid increase of the rf voltage; (e) after a quarter synchrotron period; (f) after capture in buckets of higher harmonic number.

Main Injector has the ability to operate at $\dot{\gamma} \approx 280 / \mathrm{sec}$. From Eq. (11), we get $\left(\frac{\Delta \hat{E}}{E_{\mathrm{s}}}\right)_{T} \approx \pm 0.3 \%$ for $3 \mathrm{eV} \mathrm{s}$ beam whereas the admittance is about $\pm 0.7 \%$ for the Main Injector. However, $T_{n a}+T_{n l} \approx 100 \mathrm{~m} \mathrm{~s}$, which is rather large. As a result, some longitudinal emittance dilution is expected while crossing the transition energy. An evaluation of the emittance growth is made by multiparticle beam dynamics calculations for standard transition crossing for acceleration using the $h=28 \mathrm{rf}$ system as explained below. At $27 \mathrm{GeV}$ each $2.5 \mathrm{MHz}$ bunch is transferred to a matched $53 \mathrm{MHz}$ bucket and accelerated to $150 \mathrm{GeV}$.

\section{SIMULATION OF BEAM HARMONIC TRANSFER AND ACCELERATION}

The ESME code [19] is used for the beam dynamics simulations for the antiproton acceleration scheme presented here. This program is widely used to understand the longitudinal beam dynamics at various synchrotrons at Fermilab and elsewhere. This is a turn-by-turn twodimensional tracking code which follows the evolution of a distribution of particles in longitudinal phase space, i.e., in energy and phase coordinates, as the particles are acted upon by the rf field. The single particle equations for the longitudinal motion in a synchrotron are formulated as a pair of first order nonlinear difference equations; one gives the energy change at the gap [see Eq. (3)], and the other gives the phase slip between a particle and the synchronous reference particle during transit between the rf gaps [see Eq. (4)]. For the $k$ th turn,

$$
\begin{aligned}
& E_{i, k}=E_{i, k-1}+e\left[V\left(\phi_{s, k}+h \vartheta_{i, k}\right)-V\left(\phi_{s, k}\right)\right] \\
& \phi_{i, k}-\phi_{i, s}=\vartheta_{i, k}=\frac{\tau_{s, k-1}}{\tau_{s, k}} \vartheta_{i, k-1}+2 \pi\left(\frac{\tau_{i, k}}{\tau_{s, k}}-1\right) .
\end{aligned}
$$

The subscripts $i$ and $s$ label the quantities related to particle $i$ and to the synchronous particles, respectively. The $k$ th energy increment is at the end of the $k$ th turn. For convenience in modeling, $\vartheta_{i}$ is taken as the $h=1$ phase when the synchronous particle is at the gap ( $\vartheta=0$ degree). Therefore, $-\pi \leq \vartheta_{i} \leq \pi . \tau_{s, k}=1 / 2 \pi \omega_{s, k}=C_{s, k} / \beta_{s, k} c$ is the synchronous beam circulation period. The coupling between longitudinal and transverse dynamics enters into the model through $\tau_{i, k}$. The quantity $\frac{\tau_{i, k}}{\tau_{s, k}}$ is related to $\eta$ by

$$
\frac{\tau_{i, k}}{\tau_{s, k}} \approx\left[1+\eta \beta_{s}^{2} \frac{\Delta E}{E_{s}}\right]_{k}
$$

The relation between the synchronous beam circulation period $\tau_{s, k}$ and that of the $i$ th particle $\tau_{i, k}$ is treated exactly. Thus, the treatment of the nonlinear kinematics is exact; this feature is very important when the synchronous energy is close to the transition energy.

In ESME the lattice nonlinearity is expressed as dependence of $\gamma_{T}$ on the momentum difference $\frac{\Delta p_{i}}{p_{s}}$ according to [24]

$$
\frac{C_{i}-C_{s}}{C_{s}}=\frac{\Delta C_{i}}{C_{s}}=\alpha_{0} \frac{\Delta p_{i}}{p_{s}}\left[1+\alpha_{1} \frac{\Delta p_{i}}{p_{s}}+\mathrm{O}\left(\frac{\Delta p_{i}}{p_{s}}\right)^{2}\right] .
$$

In writing Eq. (15), we have omitted the subscript $k$. This equation takes into account the first order dependence of the momentum compaction on $\frac{\Delta p}{p_{s}}$. The higher order dependences are neglected because they are very small.

Including the single particle and collective effects in simulations is quite important in understanding the high intensity beam behavior in an accelerator. In the simulation, the interaction of a beam particle with others through 
a direct particle-particle force and through the wakefield excited in the beam pipe, $\mathrm{rf}$ cavities and bellows, etc. is handled using a phenomenological model. The action of the beam on an individual particle appears as an additional accelerating or decelerating voltage acting on each particle each turn. The constant of proportionality between beam current and the voltage that beam particles experience is the longitudinal coupling impedance; it is complex and frequency dependent. The beam current is not a pure harmonic AC current. Consequently, the net voltage on a particle is calculated by evaluating that generated by each Fourier component of the beam and summing them. Thus,

$$
e V_{i, k}=\frac{N e^{2} \omega_{s, k}}{2 \pi} \sum_{m} \Lambda_{m, k} Z_{m} \exp \left\{j\left(m \vartheta_{i, m}+\psi_{m, k}+\chi_{m}\right)\right\}
$$

where $\Lambda_{m, k}$ and $Z_{m}$ are Fourier amplitudes and complex impedances, respectively. The quantities $\psi_{m, k}$ and $\chi_{m}$ are phase angles. $N$ is the total number of beam particles in the synchrotron.

In the studies carried out here, the following collective effects are taken into account: (i) space charge, (ii) reaction of the beam environment (e.g., beam pipe) on the beam distribution, and (iii) high- $Q$ rf cavity beam loading. The space charge and the coupling to the beam environment are treated in the frequency domain. Following the approach of Neil and Sessler [27], the beam is treated as a circular cylinder of charge with density independent of radius out to a fixed radius $a$ but varying along the longitudinal direction. The beam pipe is modeled as a perfectly conducting cylinder of radius $b$. For noncylindrical beam and beam pipes, we have modeled equivalent quantities. The bunch length is assumed to be far greater than $a$. The impedance $Z(\omega)$ seen by a Fourier component of the beam current at frequency $\omega / 2 \pi$ is

$$
\frac{Z(\omega)}{n}=-j \frac{Z_{0}[1+2 \ln (b / a)]}{2 \beta \gamma^{2}}+\frac{Z_{W}}{n}+\frac{Z_{\|}(\omega)}{n},
$$

where $Z_{0}=377 \Omega$ (impedance of free space). The first term represents the self-impedance arising from space charge. $Z_{W}$ is the total wall impedance of the beam pipe. $Z_{\|}$is the longitudinal coupling impedance of resonant structures, approximated by simple resonance

$$
Z_{\|}(\omega)=\frac{R_{\text {shunt }}}{1+j Q\left[\frac{\omega_{c}}{\omega}-\frac{\omega}{\omega_{c}}\right]} .
$$

For quality factor $Q=1$, Eq. (18) can be used to model the broadband wall impedance.

Very close to transition, $\eta$ approaches zero. Therefore, even a very small correction to $\gamma_{T}$ according to Eq. (15) becomes a sensitive parameter influencing the longitudinal beam dynamics near transition. Radial corrections were modeled in the simulations by making a small energy change to the beam centroid. This adjustment is needed immediately after transition.

Two types of cavity beam-loading compensation (BLC) have been taken into account in the simulation viz. feedforward BLC and feedback BLC for the rf systems. The physical effects of the feedback BLC and the feed-forward BLC are to reduce the $Q$ of the cavity and the beamloading voltage, respectively. In the simulation, the BLC is accounted for by using reduced effective $Q$ of the cavities and effective charge in a bunch.

The simulations have been carried out for acceleration of four bunches from 9 to $150 \mathrm{GeV}$ for the scheme presented here for the Main Injector using the parameters listed in Table I (and the momentum versus time curve shown in Fig. 6). Each bunch is populated using an elliptical distribution in $(\Delta E, \Delta \phi)$ phase space at $9 \mathrm{GeV}$. (This is quite a good representation of the antiproton bunches at injection into the Main Injector.) The simulations show that the 2.5 and $53 \mathrm{MHz}$ rf cavity beam-loading compensation is essential. All the simulation results presented here are for the case of two quarter rotations.

Figures 3(a)-3(d) show simulations without BLC for $6 \times 10^{10}$ particles/bunch with $\varepsilon_{l}=1.3 \mathrm{eV} \mathrm{s}$ (for $95 \%$ of the bunch). Considerable phase-space distortion is observed in about $0.5 \mathrm{sec}$ after injection [see, e.g., Fig. 3(b)], before acceleration commences. At $27 \mathrm{GeV}$ the centroid of the first bunch is found to be shifted by $>180^{\circ}$ of $h=588 \mathrm{rf}$ (for the rest this shift was larger, not shown here). Figures $3(\mathrm{e})-3(\mathrm{~h})$ show simulation results with BLC for intensity of $17 \times 10^{10}$ particles/bunch, approximately a factor of 3 more bunch intensity than that used for Figs. 3(a)-3(d). For these simulations we have assumed about a factor of 5 reduction in $Q$ for the cavities and a factor of 10 reduction in charge to account for feedback and feed-forward BLC, respectively. A total of about $30 \%$ emittance growth is predicted from 9 to $150 \mathrm{GeV}$. Out of this less than $10 \%$ emittance growth is during beam acceleration including the transition crossing. The rest is seen during harmonic transfer; we attribute this growth to the shape mismatch while transferring the bunch from the $h=28$ to the $h=588$ bucket. The mismatch is expected to be more on the fourth bunch because of beam loading [e.g., see Fig. 4(c)]. Nevertheless, the overall acceleration efficiency found to be $100 \%$.

Figures 4(a) and 4(b) show the predicted transient residual beam-loading voltage on the cavities during the entire acceleration cycle with BLC. Increased line charge density is observed during transition crossing, harmonic transfer, and during $53 \mathrm{MHz}$ acceleration from 27 to $150 \mathrm{GeV}$. Consequently, the transient beam-loading voltage display increased values at that time in the acceleration cycle. Calculations showed that the residual beam-loading voltage on the $53 \mathrm{MHz}$ system is about $2.1 \mathrm{kV}$ at $17 \times 10^{10}$ particles/bunch during transition crossing, which is con- 
TABLE I. Fermilab Main Injector parameter list used for the numerical tracking.

\begin{tabular}{|c|c|}
\hline $\begin{array}{l}\text { Mean radius of Main Injector } \\
\text { Nominal } \gamma_{T} \\
\text { Beam momentum }{ }^{\mathrm{a}} \\
(d p / d t)_{\max } \text { for } 8-27 \mathrm{GeV} \text { acceleration with } 2.5 \mathrm{MHz} \mathrm{rf} \\
(d p / d t)_{\max } \text { for } 27-150 \mathrm{GeV} \text { acceleration with } 53 \mathrm{MHz} \mathrm{rf}\end{array}$ & $\begin{array}{l}528.3 \mathrm{~m} \\
21.8 \\
8.9-149.6 \mathrm{GeV} / c \\
3.2 \mathrm{GeV} / c / \mathrm{s} \\
120.0 \mathrm{GeV} / c / \mathrm{s}\end{array}$ \\
\hline $\begin{array}{l}\text { Maximum available rf voltage: } \\
2.5 \mathrm{MHz} \text { rf system }(h=28) \\
5 \mathrm{MHz} \text { rf system }(h=56) \\
53 \mathrm{MHz} \text { rf system }(h=588)\end{array}$ & $\begin{array}{l}75 \mathrm{kV} \\
15 \mathrm{kV} \\
\approx 2 \mathrm{MV} \text { (close to } 8 \mathrm{GeV}) \\
4 \mathrm{MV} \text { (elsewhere) }\end{array}$ \\
\hline $\begin{array}{l}\text { Pbar bunch properties at injection: } \\
\text { Longitudinal emittance per bunch } \\
\text { Bunch intensity per } 2.5 \mathrm{MHz} \text { bucket } \\
\text { Number of bunches/transfer }\end{array}$ & $\begin{array}{l}0.8-2.6 \mathrm{eVs} \\
6-17\left(\times 10^{10}\right) \\
4\end{array}$ \\
\hline $\begin{array}{l}\text { Space charge simulations: } \\
Z / n \text { (broadband) } \\
\text { Average beam pipe radius } \\
\alpha_{0} \alpha_{1} \\
\text { Average beam radius } \\
\text { Beam pipe cutoff frequency }\end{array}$ & $\begin{array}{l}1.6-4.8 \Omega \\
5.1 \mathrm{~cm} \\
0.0021 \\
0.5 \mathrm{~cm} \\
1.7 \mathrm{GHz}\end{array}$ \\
\hline $\begin{array}{l}\text { Beam-loading compensation simulations: } \\
2.5 \mathrm{MHz} \text { rf system (number of rf cavities }=5 \text { ) } \\
\text { Shunt impedance and } Q \\
\text { Effective reduction in } Q \text { (feedback compensation) } \\
\text { Effective reduction in charge (feed-forward comp.) }\end{array}$ & $\begin{array}{l}R=45 \mathrm{k} \Omega / \text { cavity and } Q=112.5 \\
\text { factor of } 5 \\
\text { factor of } 10\end{array}$ \\
\hline $\begin{array}{l}53 \mathrm{MHz} \text { rf system (number of rf cavities }=18) \\
\text { Shunt impedance and } Q \\
\text { Effective reduction in } Q \text { (feedback compensation) } \\
\text { Effective reduction in charge (feed-forward compensation) }\end{array}$ & $\begin{array}{l}R=520 \mathrm{k} \Omega / \text { cavity and } Q=5000 \\
\text { factor of } 5 \\
\text { factor of } 10\end{array}$ \\
\hline
\end{tabular}

${ }^{\mathrm{a}}$ Here the beam momenta are quoted to first decimal place and the corresponding momentum ramp is shown in Fig. 6 . The beam energy is given by $E=\sqrt{p^{2} c^{2}+m_{p}^{2} c^{4}}$, where $m_{p}$ is the rest mass of proton or antiproton. We quote round figures for beam energy in the text.

sistent with the measured value of $1.0 \mathrm{kV}$ at $8 \times 10^{10}$ particles/bunch. A dip in beam-loading voltage is seen at about $7.2 \mathrm{sec}$ in acceleration cycle; this corresponds to the end of the first bunch rotation in the $2.5 \mathrm{MHz}$ rf bucket [e.g., Fig. 3(g)] with maximum bunch length. Figure 4(c) shows the instantaneous residual beam-loading voltage buildup as the four bunches pass by the $53 \mathrm{MHz}$ rf cavities during harmonic transfer. The beam-loading voltage decay time constant is about $2.85 \mu \mathrm{s}$.

Figure 5(a) shows the predicted average longitudinal emittance dilution as a function of initial bunch intensity for different initial $\varepsilon_{l}$. The maximum emittance growth is seen on the fourth bunch. Simulations for $2.2 \leq \varepsilon_{l} \leq$ $2.6 \mathrm{eV}$ s showed less than $3 \%$ beam loss. Figure 5(b) shows a comparison of emittance growth during acceleration from 9 to $150 \mathrm{GeV}$ between the first and the last bunch in a train of four bunches for $\varepsilon_{l}=1.3 \mathrm{eV} \mathrm{s}$. The emittance growth averaged over four bunches is about $50 \%$ for intensity $\sim 17 \times 10^{10}$ particle/bunch; no beam particle loss is seen. The causes of beam particle losses for $\varepsilon_{l} \geq$ $2.2 \mathrm{eV} \mathrm{s}$ originate primarily during transition crossing. For example, in the case of $\varepsilon_{l} \approx 2.5 \mathrm{eV} \mathrm{s}$, the nonlinear effects during transition crossing give rise to distorted phase-space distribution of beam particles. This caused about $3 \%$ of the beam particles to form a tail on the bunch. During harmonic transfer they ended up outside the $53 \mathrm{MHz}$ rf bucket forming DC beam in the accelerator, getting lost at the beginning of $27-150 \mathrm{GeV}$ acceleration. This phenomenon is found to have weak dependence on the bunch intensity. On the other hand, in the case of high intensity $0.9 \mathrm{eV} \mathrm{s}$ bunches, there is emittance growth during transition crossing and bucket mismatch during harmonic transfer. These two phenomena led to an overall emittance growth of about $80 \%$ for the highest intensity bunches studied here. However, no particle loss is seen throughout the acceleration for the low emittance cases. To achieve an average emittance dilution less than $15 \%$ for $\varepsilon_{l}=1.3 \mathrm{eV} \mathrm{s}$ with $17 \times 10^{10}$ particle/bunch, the required feed-forward BLC 


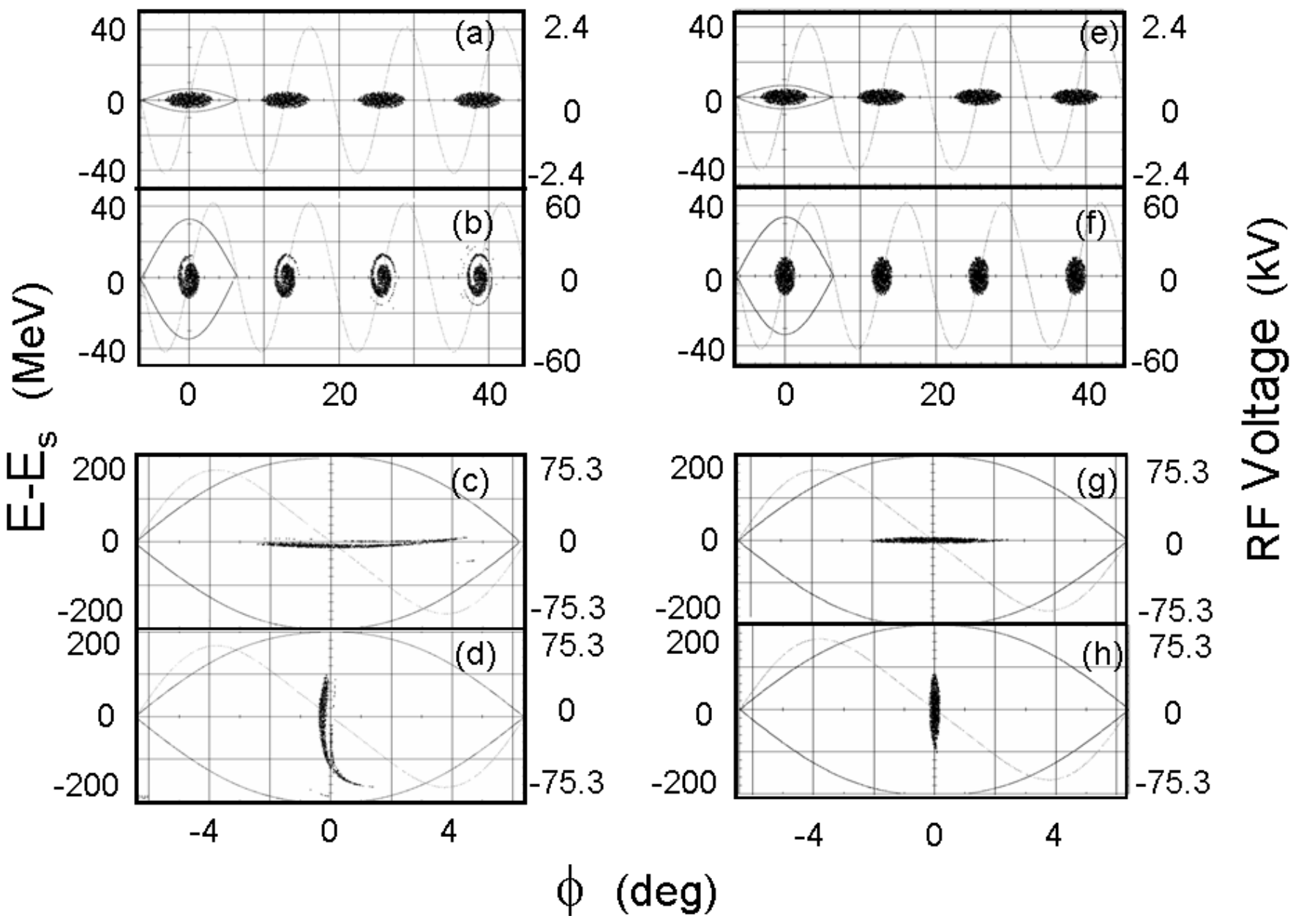

FIG. 3. Simulated $(\Delta E, \Delta \phi)$-phase distribution of antiprotons without (left column) and with (right column) BLC for $1.3 \mathrm{eV} \mathrm{s}(95 \%)$ bunches. (a) Four bunches of $6 \times 10^{10}$ particles/bunch at $9 \mathrm{GeV}$ immediately after the injection. (b) A few seconds after injection, still at $9 \mathrm{GeV}$. (c) Beam at $27 \mathrm{GeV}$ before bunch rotation (first bunch only) and (d) after bunch rotation (first bunch only). This clearly shows significant bunch mismatch and one expects significant emittance growth. The simulations with BLC for $17 \times 10^{10}$ particles/bunch shown in (e)-(h) indicate little phase-space distortion.

is predicted to be about a factor of 3 more than used in these simulations.

\section{EXPERIMENT AND DISCUSSIONS}

The experiment is conducted in the Fermilab Main Injector with a proton beam. Four consecutive $2.5 \mathrm{MHz}$ bunches separated by about $398 \mathrm{~ns}$ are prepared [28] at $9 \mathrm{GeV}$ using four groups of $53 \mathrm{MHz}$ bunches, each containing about 5 to 13 bunches of protons from the Fermilab Booster. The experiment is carried out for intensities in the range of $(3-12) \times 10^{10}$ protons $/ 2.5 \mathrm{MHz}$ bunch and $\varepsilon_{l} \sim$ 1-3 eVs. The properties of the $2.5 \mathrm{MHz}$ bunches are chosen to mimic the antiproton bunches from the Recycler or the Accumulator. Both 2.5 and $53 \mathrm{MHz}$ beam-loading compensation [29] are used for the beam studies.

During the $2.5 \mathrm{MHz}$ acceleration from 9 to $27 \mathrm{GeV}$, the $53 \mathrm{MHz}$ rf power amplifiers are turned off while keeping the $53 \mathrm{MHz}$ feed-forward beam-loading compensation active. Acceleration is performed with two radial and phase control systems [30]; one controls the $2.5 \mathrm{MHz}$ rf acceleration from 9 to $27 \mathrm{GeV}$ and another controls the $53 \mathrm{MHz}$ rf acceleration from 27 to $150 \mathrm{GeV}$. These control systems are turned off during bunch rotation and harmonic transfer. The first rotation is performed with $2.5 \mathrm{MHz} \mathrm{rf}$ voltage held at $4 \mathrm{kV}$ and the second rotation at $\sim 60 \mathrm{kV}$. During the second rotation about $10.8 \mathrm{kV}$ of $5 \mathrm{MHz} \mathrm{rf}$ component is added, with a phase offset of $180^{\circ}$ relative to the $2.5 \mathrm{MHz}$ rf wave, to increase the extent of the linear region of the total $\mathrm{rf}$ voltage wave form. A peak detection device is used as a diagnostic tool to monitor the peak current in the Main Injector during bunch rotation. The $53 \mathrm{MHz}$ cavities are turned on with a matched rf voltage at the exact moment of maximum current in the peak detector. The matching voltage is found to be in the range of 0.5 to $0.7 \mathrm{MV}$ on the $53 \mathrm{MHz}$ rf system, depending on the longitudinal emittance of the beam. 

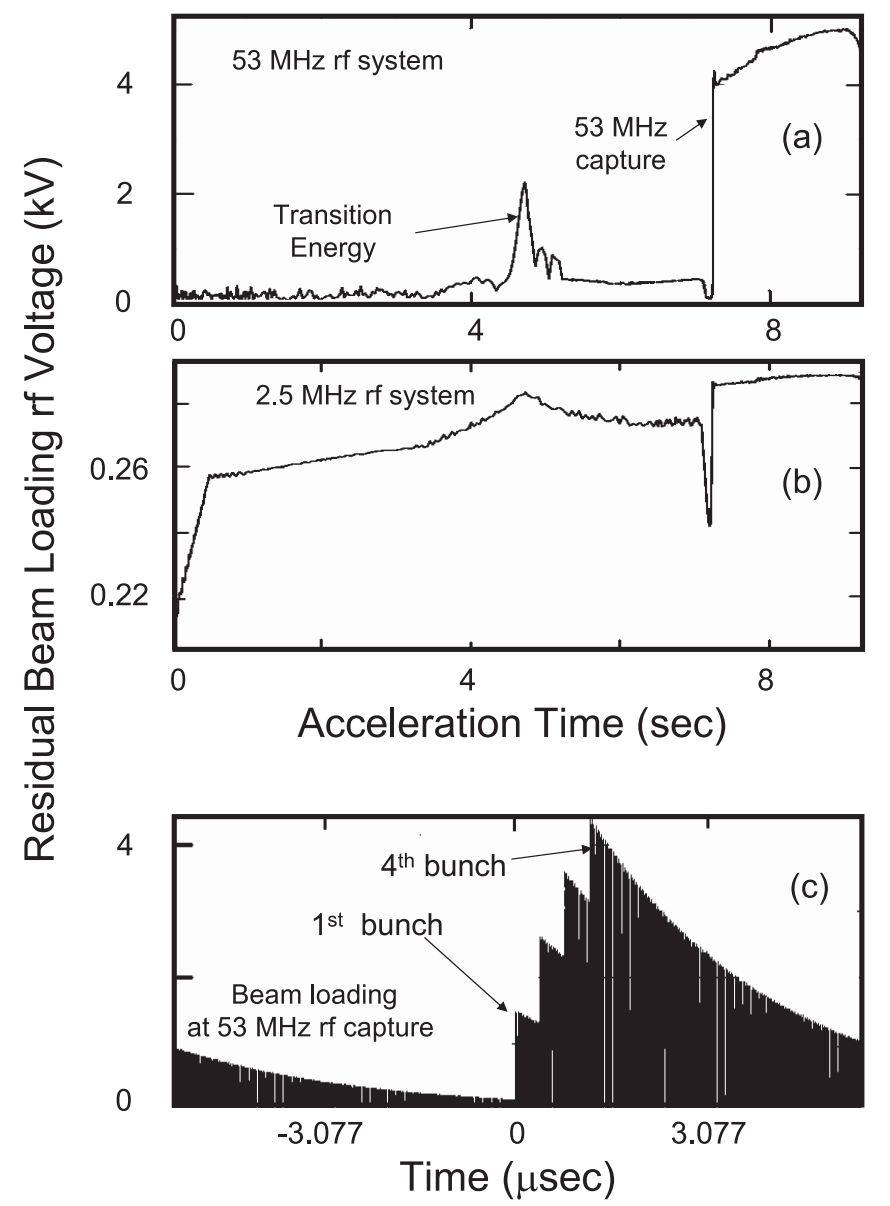

FIG. 4. Predicted residual beam-loading voltage for four bunches with $17 \times 10^{10}$ particles/bunch for (a) $53 \mathrm{MHz}$ rf cavities, (b) $2.5 \mathrm{MHz}$ rf cavities for the duration of beam acceleration from 9 to $150 \mathrm{GeV}$. The $9-150 \mathrm{GeV}$ acceleration time was about $9.3 \mathrm{sec}$. (c) Instantaneous $53 \mathrm{MHz}$ residual beamloading voltage at its maximum during harmonic transfer. The simulations are carried out with 2.5 and $53 \mathrm{MHz}$ rf cavity BLC which include both feedback and feed-forward BLCs as explained in the text and Table I. The transition time and the time of transfer between rf systems are also indicated on (a). The steps in (c) correspond to the passing of each bunch. The $\varepsilon_{l}$ in this case was $1.3(95 \%) \mathrm{eVs}$.

Figure 6 shows the measured 9 to $150 \mathrm{GeV}$ magnet ramp, the peak voltages for the two rf systems, and the total beam intensity for four $2.5 \mathrm{MHz}$ bunches. The sharp dip and rise of the $2.5 \mathrm{MHz}$ rf voltage curve (red) around $8.5 \mathrm{sec}$ in the cycle are, respectively, associated with the first and the second quarter rotations during harmonic transfer. Data shown are obtained with accelerator parameters optimized for $\sim 6 \times 10^{10}$ particle/bunch at $\varepsilon_{l} \approx$ $2.0 \mathrm{eV}$ s. The $2.5 \mathrm{MHz}$ bunch intensities have been changed by increasing the number of $53 \mathrm{MHz}$ bunches and/or increasing the $53 \mathrm{MHz}$ bunch intensities of protons used in their preparation in the Main Injector. Consequently, the longitudinal emittances were also different.

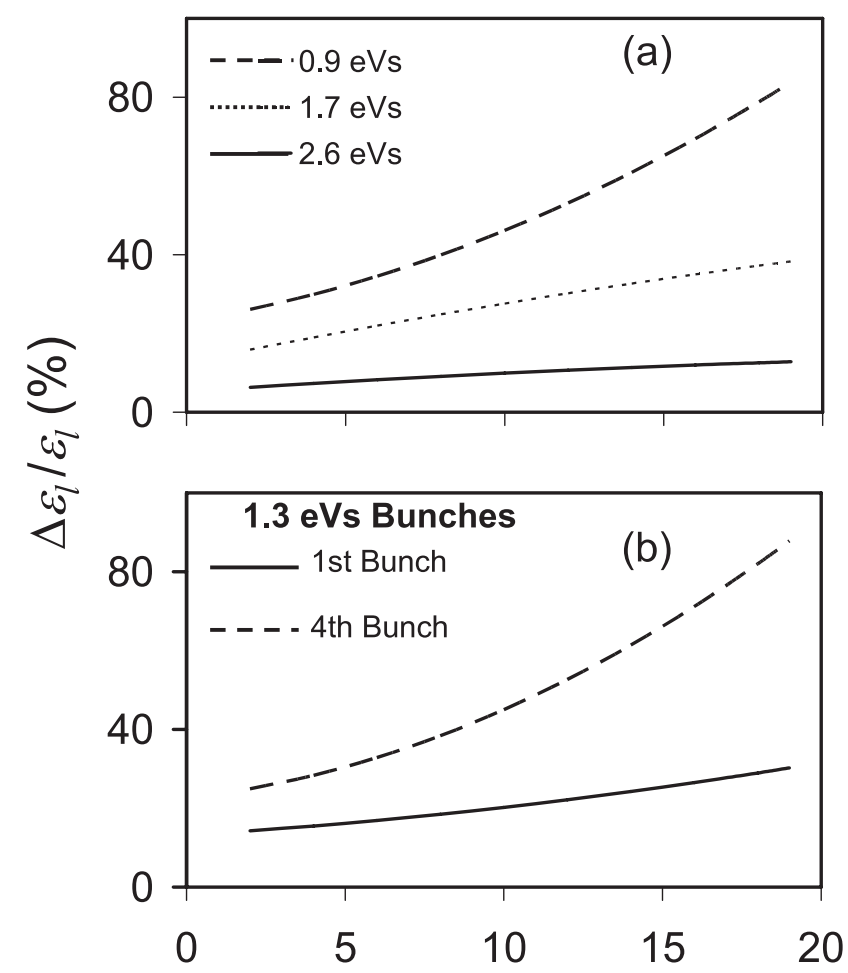

Number of Antiprotons/2.5 MHz Bunch (x1010)

FIG. 5. The predicted $\varepsilon_{l}(95 \%)$ growth as a function of antiproton bunch intensity from ESME simulations for beam acceleration from 9-150 GeV. Calculations are carried out including BLC as in Table I. (a) Average $\varepsilon_{l}$ growth for $\varepsilon_{l}(95 \%)=0.9,1.7$, and $2.6 \mathrm{eV} \mathrm{s}$ bunches. (b) $\varepsilon_{l}$ growth for the first bunch and the last bunch in the case of $\varepsilon_{l}($ initial $)=1.3 \mathrm{eV} \mathrm{s}$.

The $\varepsilon_{l}$ of the beam has been measured at 9,27 , and $150 \mathrm{GeV}$ using a wide band resistive wall pickup monitor and a Direct Current Current Transformer for beam intensity. For the data shown in Fig. 6, the measured $\varepsilon_{l}$ at injection were about $1.0 \pm 0.2,2.0 \pm 0.4$, and $2.3 \pm 0.5$ with $3.65,6.65$, and $8.25 \times 10^{10}$ particle/bunch, respectively. The general features of beam acceleration as a function of beam intensity and longitudinal emittance are in good agreement with our simulations. For example, in the case of $2 \mathrm{eV} \mathrm{s}$ bunches, we expect injection to extraction efficiency to be nearly $100 \%$. On the other hand, the $2.3 \mathrm{eV} \mathrm{s}$ bunches are expected to show $100 \%$ acceleration efficiency from 9 to $27 \mathrm{GeV}$, but about $2.5 \%$ of the particles outside of the $53 \mathrm{MHz}$ rf bucket due the combination of nonlinear effects at transition and filamentation during bunch rotation. These particles get lost at the beginning of the $27-150 \mathrm{GeV}$ acceleration, in good agreement with the simulations.

Figure 7 shows a typical mountain range plot of the rotations and the harmonic transfers from $2.5 \mathrm{MHz}$ rf buckets to $53 \mathrm{MHz}$ buckets for the first bunch and its comparison with ESME prediction. The bunch intensity 


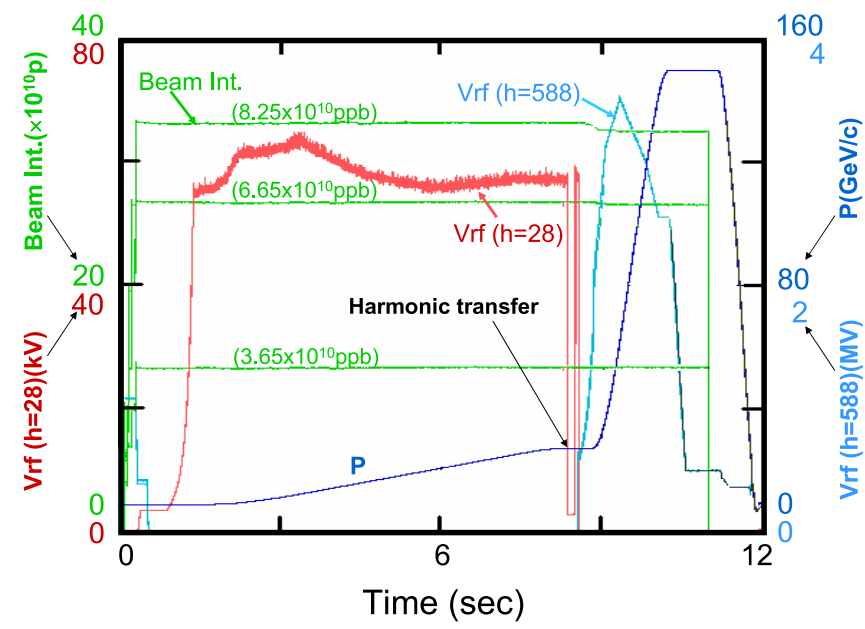

FIG. 6. (Color) Experimental data for the acceleration scheme for three different beam intensities. 8.9-149.6 GeV/c momentum ramp (P, blue), $2.5 \mathrm{MHz}$ peak rf voltage [Vrf $(h=28)$, red], $53 \mathrm{MHz}$ peak rf voltage [Vrf $(h=588)$, cyan], and the beam intensity [Beam Intensity, green] versus acceleration cycle time. Four $2.5 \mathrm{MHz}$ bunches are accelerated from 9 to $150 \mathrm{GeV}$. The 2.5 MHz bunch intensities for three cases are shown. The longitudinal emittances of the $2.5 \mathrm{MHz}$ bunches at $9 \mathrm{GeV}$ were about 1.0, 2.0, and $2.3 \mathrm{eV} \mathrm{s}$ (with an error of $\sim 20 \%$ ). The acceleration efficiency found to be in the range of $97.5 \%-$ $100 \%$.

was about $6 \times 10^{10}$ particle/bunch. In this case, the measured $\varepsilon_{l}$ at the beginning and end of the harmonic transfer are $(2.0 \pm 0.3) \mathrm{eV} \mathrm{s}$ and $2.3 \pm 0.3 \mathrm{eV} \mathrm{s}$, respectively. No emittance growth is seen during the $27-150 \mathrm{GeV}$ acceleration. Studies showed that the exact timing of the $53 \mathrm{MHz}$ capture (with a precision better than $0.5 \mathrm{~m} \mathrm{~s}$ ) is critical to obtain minimum emittance dilution. The optimum bunch rotation time is a function of bunch intensity and $\varepsilon_{l}$ of the bunch. As the bunch intensity is increased at nearly constant $\varepsilon_{l}$, the transient beam-loading voltage goes up. Consequently, cavity voltage seen by bunches decrease, resulting in longer rotation time. Similarly, for fixed bunch intensity, if the $\varepsilon_{l}$ is varied, the bunches with larger $\varepsilon_{l}$ take a longer rotation time.

Since the acceleration scheme is rather simple, the beam is less susceptible to emittance dilution. Nonetheless, longitudinal emittance dilution is expected mainly at two points in the entire acceleration cycle, one at transition crossing and another at harmonic transfer. Because of the relatively long transition crossing interval, there is always a certain amount of beam mismatch to the bucket after transition crossing. In the present experiment, the oscillation of the bunch centroid is observed after transition crossing which led to $\varepsilon_{l}$ growth in the range of $10 \%-$ $30 \%$ depending upon the initial longitudinal emittance and bunch intensity. This emittance dilution can be controlled using a longitudinal bunch by bunch digital damper [31] and special transition crossing schemes [22,23].

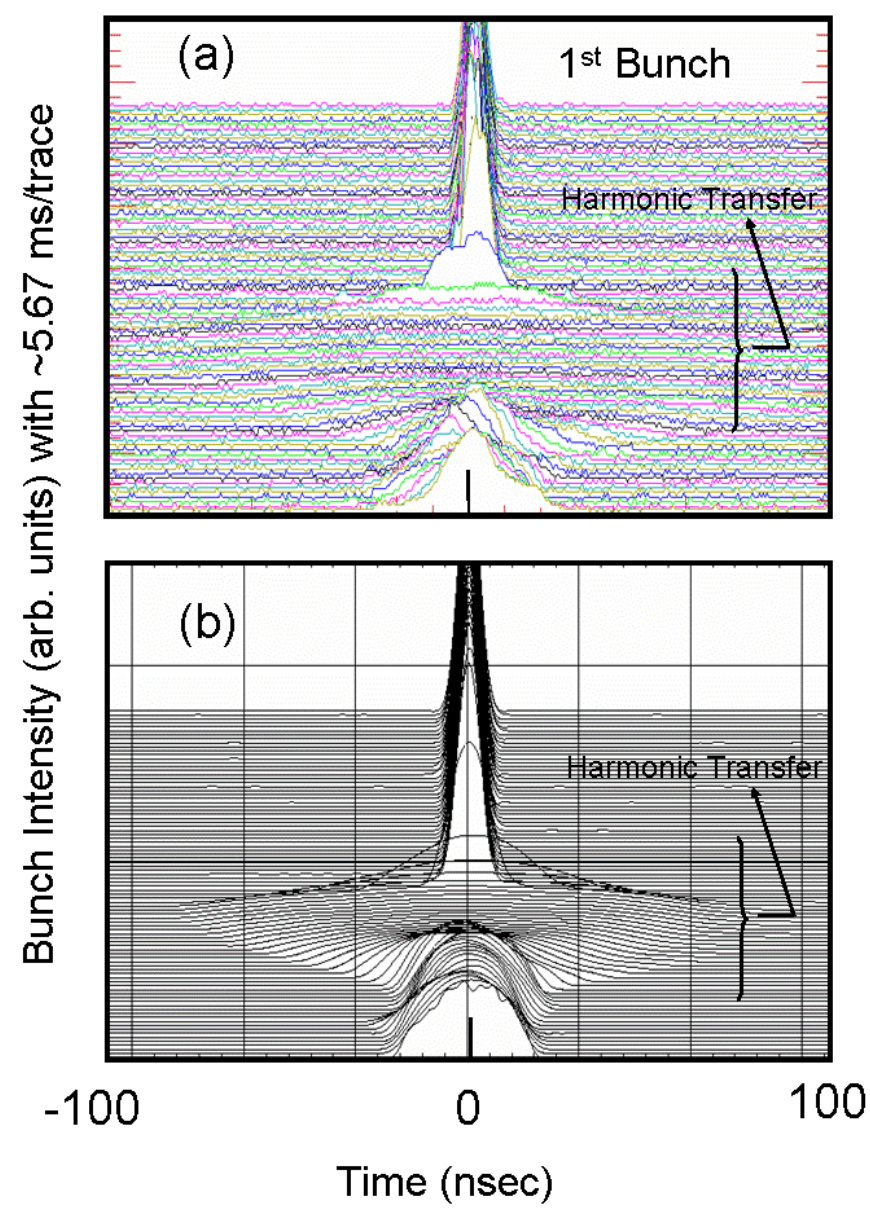

FIG. 7. (Color) Mountain range plots during harmonic transfer from a $2.5 \mathrm{MHz}$ bucket to a $53 \mathrm{MHz}$ bucket at $27 \mathrm{GeV} / c$ for $6 \times 10^{10} /$ bunch and $\varepsilon_{l}(95 \%)=2 \mathrm{eV} \mathrm{s}$ : (a) experimental results and (b) ESME predictions for the first bunch from a train of four bunches. The total time along the vertical axes is about $0.5 \mathrm{sec}$.

Experimentally, we have shown nearly $100 \%$ acceleration efficiency from 9 to $150 \mathrm{GeV}$ for $2.5 \mathrm{MHz}$ beam with $\leq$ $6.6 \times 10^{10}$ particle/bunch and longitudinal emittance dilution less than $30 \%$ for $\varepsilon_{l} \sim 2 \mathrm{eV} \mathrm{s}$ even without the dampers or any special transition crossing schemes.

The transverse emittances of the beam are measured using the flying wires [32] at various times up the ramp. Data showed $\leq 1 \pi$-mm-mr emittance growth from 9 to $150 \mathrm{GeV}$.

Recently, we have conducted test experiments using an antiproton beam from the Recycler with $\varepsilon_{l} \sim$ $1 \mathrm{eV} \mathrm{s} / 2.5 \mathrm{MHz}$ bunch but at low intensity $(\sim 1.8 \times$ $10^{10}$ antiproton/bunch). The results are consistent with proton measurements.

\section{SUMMARY}

Currently, the Fermilab Tevatron is the highest energy hadron collider in the world. One of the prime limiting factors to increased luminosity are preserving the longitu- 
dinal emittance and beam loss for the antiproton bunches. The current antiproton acceleration and coalescing scheme in the Main Injector is responsible for more than $10 \%$ beam loss and more than a factor of 2 longitudinal emittance growth. Elimination of this beam loss and emittance dilution helps reach (and go beyond) the design goal for proton-antiproton luminosity of the Tevatron. ${ }^{2}$

We have developed and demonstrated a viable scheme for acceleration of antiproton beam bunches using two different $\mathrm{rf}$ systems and an $\mathrm{rf}$ harmonic transfer at an intermediate energy. The technique is quite general and might be adopted at other accelerator facilities. The scheme has been studied with multiparticle beam dynamics simulations and beam experiments in the Main Injector. Simulations predict that for $\varepsilon_{l} \leq 2.2 \mathrm{eV} \mathrm{s}$ and intensity $\sim 2-17 \times 10^{10}$ antiprotons/bunch, one can achieve no beam loss in the Main Injector for acceleration from 9$150 \mathrm{GeV}$ with the available beam-loading compensation for 2.5 and $53 \mathrm{MHz}$ rf systems. We can achieve less than $50 \%$ emittance dilution for $\varepsilon_{l}$ in the range of $1.2-2.2 \mathrm{eV} \mathrm{s}$ and less than $75 \%$ for $\varepsilon_{l} \sim 0.9-1.2 \mathrm{eV} \mathrm{s}$. Experiments with intensity in the range of $(3-12) \times 10^{10}$ particle $/ 2.5 \mathrm{MHz}$ bunch confirm our simulation results. With the proposed scheme for the antiproton acceleration in the Main Injector one can gain in the peak proton-antiproton luminosity at the Tevatron by $\geq 15 \%$.

In principle, one can reduce the longitudinal emittance dilution related to the transition crossing by increasing $\dot{\gamma}$ through the transition (i.e., by reducing $T_{n a}+T_{n l}$ ). This needs upgrades to the $2.5 \mathrm{MHz}$ rf system in the Main Injector. Similarly, with more beam-loading compensation in the Main Injector, the growth in $\varepsilon_{l}$ can be reduced further.

Results from the low intensity antiproton beam experiments from the Recycler are consistent with proton beam measurements. Implementation of this scheme for Tevatron collider operation is in progress.

As a final note, we expect that the applications of the technique described here for beam acceleration need not be limited to just two rf systems. It would be possible to use more rf systems at higher harmonics as the energy of the beam is increased in the synchrotron.

\section{ACKNOWLEDGMENTS}

We would like to thank B.E. Chase, J.E. Dey, P.W. Joireman, and K. Meisner for their help in developing necessary rf hardware and software tools used in the

\footnotetext{
${ }^{2}$ Since the submission of the paper, the Tevatron protonantiproton luminosity has been steadily increased to $2.92 \times$ $10^{32} / \mathrm{cm}^{2} / \mathrm{sec}$ by increasing the antiproton bunch intensity (with the bunch coalescing scheme in use). This is slightly over the run II peak luminosity goal. However, additional $15 \%$ increase in the peak luminosity is expected with the new scheme discussed in this paper.
}

beam experiments, and D. Capista and P. Adamson for their help during the beam studies. C. Bhat would like to thank Pushpalatha Bhat for her comments on this paper and Shreyas Bhat for careful editing of the paper. We acknowledge the support of the U. S. Department of Energy. Fermi National Accelerator Laboratory is operated by the Universities Research Association, under contract with the U.S. Department of Energy.

[1] Fermi National Accelerator Laboratory, Batavia, Illinois, operated by Universities Research Association Inc., under contract with the U.S. Department of Energy, 1984, http:// library.fnal.gov/archive/design/fermilab-design-1984-01. shtml.

[2] J.E. Griffin, J.A. MacLachlan, and Z. B. Qian, IEEE Trans. Nucl. Sci. NS-30, 2627 (1983).

[3] CERN, Geneva CERN/PS/AA 78-3 27.1.1978.

[4] C.M. Bhat, MI Note 268 (2000) (unpublished), http:// beamdocs.fnal.gov/DocDB/0003/000362/001/minote268. pdf; C. M. Bhat, B. C. Brown, D. Capista, A. Hahn, D. E. Johnson, I. Kourbanis, S. Mishra, S. M. Pruss, G. Wu, M.-J. Yang (FNAL), and S. Assadi (ORNL), Proceedings of Particle Accelerator Conference, Chicago, IL, 2001 (IEEE, Piscataway NJ, 2001) p. 1705.

[5] J. Dey, I. Kourbanis, and D. Wildman, Proceedings of Particle Accelerator Conference, Dallas, TX, 1995 (IEEE, Piscataway NJ, 1995) p. 3312.

[6] C. M. Bhat, D. Capista, B. Chase, J. E. Dey, I. Kourbanis, K. Seiya, and V. Wu, Proceedings of Particle Accelerator Conference, Knoxville, Tennessee, 2005 (IEEE, Piscataway, NJ, 2005), p. 1763.

[7] Fermilab Internal Report, 1994 (unpublished), http:// www.fmi.fnal.gov/fmiinternal/MI_Technical_Design / index.html.

[8] D. A. Edwards and M. J. Syphers, An Introduction to the Physics of High Energy Acelerators, 1st edition (Wiley \& Sons, Inc., New York, 1993).

[9] CERN/AC/95-05, CERN, 1995, http://ab-div.web.cern.ch/ ab-div /Publications/LHC-DesignReport.html.

[10] P. C. Bhat and W. J. Spalding, Hadron Collider Physics: 15th Topical Conference on Hadron Collider Physics, AIP Conference Proceedings 753 (AIP, New York, 2004), pp. 30-41.

[11] "Run II Hand Book" (unpublished), http://www-bd.fnal. gov/runII/ and the current Tevatron luminosity status is available on http://www-bdnew.fnal.gov/operations/lum/ lum.html.

[12] G. Jackson, Fermilab-TM-1991, 1996 (unpublished), http://lss.fnal.gov/archive/test-tm/1000/fermilab-tm-1991. pdf.

[13] R. Garoby, IEEE Trans. Nucl. Sci. NS-32, 2323 (1985); H. Damerau and R. Garoby, Proceedings of EPAC 2004, Lucerne, Switzerland, p. 1852.

[14] J. M. Brennan, M. Blaskiewicz, J. Butler, J. DeLong, W. Fischer, and T. Hayes, Proceedings of EPAC 2004, Lucerne, Switzerland, p. 905.

[15] W. Chou, J. Griffin, J. A. MacLachlan, and S. Werkema, Proceedings of Particle Accelerator Conference, Portland, 
Oregon, 2003 (IEEE, Piscataway, NJ, 2003), p. 1554.

[16] J. Wei, Ph.D. thesis, State University of New York at Stony Brook, 1990.

[17] C. M. Bhat and J. A. MacLachlan, Proceedings of Particle Accelerator Conference, Vancouver, B.C., Canada, 1997 (IEEE, Piscataway, NJ, 1997), p. 1590; C. M. Bhat, MI Note 271 (2000) (unpublished), http://beamdocs.fnal.gov/ DocDB/0007/000742/001/MI-0271.pdf; C. M. Bhat, B. C. Brown, D. Capista, B. Chase, A. Hahn, D. E. Johnson, I. Kourbanis, K. Meisner, S. Mishra, S. M. Pruss, G. Wu, M.-J. Yang (FNAL), S. Assadi (ORNL), Proceedings of Particle Accelerator Conference, Chicago, IL, USA, 2001 (IEEE, Piscataway, NJ, 2001), p. 440.

[18] V. Wu, C. M. Bhat, B. Chase, J. Dey, J. A. MacLachlan, K. Meisner, and J. Reid, Proceedings of Particle Accelerator Conference, Portland, Oregon, 2003, Ref. [15], p. 1769; V. Wu, C. M. Bhat, B. Chase, J. E. Dey, and K. G. Meisner, Proceedings of Particle Accelerator Conference, Knoxville, Tennessee, 2005 (IEEE, Piscataway, NJ, 2005), pp. 1303 and 2164.

[19] J.A. MacLachlan, HEACC'98, XVII International Conference on High Energy Accelerators, Dubna (1998), p. 184; A. Maclachlan (private communication); The latest version of the code is available at http://www-ap.fnal.gov/ ESME/.

[20] G. Dome, CERN SPS/84-13(ARF) (1984) (unpublished).

[21] S. Y. Lee, Accelerator Physics, 1st Edition (World Scientific, Singapore, 1999), Chap. 3, p. 217, and references therein; K. Y. Ng, Fermilab-FN-0713, http:// lss.fnal.gov/archive/test-fn/0000/fermilab-fn-0713.shtml.

[22] A. Sorenssen, Part. Accel. 6, 141 (1975).

[23] C. M. Bhat, J. Griffin, J. MacLachlan, M. Martens, K. Meisner, and K. Y. Ng, Phys. Rev. E 55, 1028 (1997), and references therein.

[24] E. D. Courant and H. S. Snyder, Ann. Phys. (N.Y.) 3, 1 (1958).
[25] K. Johnsen, Proc. CERN Symp. High Energy Accel. Pion Phys. 1, 106 (1956); the parameter $\alpha_{2}$ in this paper is referred to as $\alpha_{1}$ in the text here.

[26] S. Y. Lee and J. Wei, Proceedings of the European Particle Accelerator Conference in Rome, Italy, 1988, edited by S. Tazzari (World Scientific, Singapore, 1989) Vol. 1, pp. 764-766.

[27] V. K. Neil and A. M. Sessler, Nucl. Instrum. Methods 36, 429 (1965).

[28] C. M. Bhat, MI Note 263 (2000) (unpublished), http:// beamdocs.fnal.gov/DocDB/0003/000352/001/mi-note263. pdf.

[29] J. Dey, J. Reid, and J. M. Steimel, Proceedings of Particle Accelerator Conference, Portland, Chicago, 2001 (IEEE, Piscataway, NJ, 2001), pp. 876 and 879; J. Dey, I. Kourbanis, J. Reid, and J. Steimel, Proceedings of Particle Accelerator Conference, Portland, Oregon, 2003, Ref. [15], pp. 3353 and 3356; D. Wildman and J. Dey (private communication).

[30] B. Chase, A. Mason, and K. Meisner, Proceedings of the ICALEPCS'97, Beijing, China (1997), http://www. aps.anl.gov/conferences/icalepcs/97/paper97/p230.pdf; B. Chase and K. Meisner (private communication).

[31] P. Adamson, P. Adamson, B. Ashmanskas, G. W. Foster, S. U. Hansen, A. Marchionni, D. J. Nicklaus, A. Semenov, and D. Wildman, Proceedings of Particle Accelerator Conference, Knoxville, TN, 2005 (IEEE, Piscataway, NJ, 2005), p. 1440.

[32] A. Barisy, R. Bellone, R. Blanchard, J. Camas, L. Evans, G. Ferioli, R. Hopkins, P. Sievers, and J. P. Zanasco, IEEE Trans. Nucl. Sci. NS-28, 2180 (1981), and references therein; J. Gannon, C. Crawford, D. Finley, R. Flora, T. Groves, and M. MacPherson, Proceedings of 1989 IEEE Particle Accelerator Conference, Chicago, Illinois 1989 (IEEE, Piscataway, NJ, 1989), p. 68. 